\title{
Magnetic-grating free-induction decay and magnetic-grating echo using ultrafast excitation pulses
}

\author{
B. Dubetsky, P. R. Berman \\ Physics Department, University of Michigan, Randall Laboratories, Ann Arbor, MI 48109-1120, USA \\ (Fax: + 1-313/763-9694)
}

Received 8 November 1993/Accepted 11 May 1994

\begin{abstract}
The interaction of atoms with ultrafast, counterpropagating optical fields is considered. The magnetic degeneracy and hyperfine splitting of the atomic levels are included in the calculations, which are carried out for arbitrary polarizations of the incident fields. The counterpropagating fields produce spatial harmonics in the ground state density matrix (gratings) which can be monitored by backscattering of a traveling wave probe pulse. Two types of excitation schemes are analyzed. The Magnetic-Grating Free-Induction Decay (MGFID) consists of excitation with a single counterpropagating wave field, while the Magnetic-Grating Echo (MGE) involves excitation by two counterpropagating wave fields, separated in time by $\mathrm{T}$. The atomic response to the probe pulse is calculated in lowest-order perturbation theory for atoms cooled below the Doppler limit of laser cooling. Both the MGFID and MGE signals consist of pulses having a duration of order of the excited state lifetime, modulated at frequencies corresponding to the various hyperfine transitions. As a function of the delay between pulses, the signals oscillate at frequencies determined by the ground state hyperfine splittings. General expressions for the MGFID and MGE signals are derived and specific results are presented for the $\mathrm{D}_{2}$ line in $\mathrm{Na}$.
\end{abstract}

PACS: $42.50 . \mathrm{Md}, 42.65 . \mathrm{Re}, 32.00$

The resonance interaction of atoms with radiation fields can lead to the creation of macroscopic optical dipole coherence as well as spatially modulated ground and excited state populations and magnetic state coherences. Observation of the decay of these quantities provides information about relaxation in the medium. Monitoring of the ground state is of special interest owing to its long lifetime and high sensitivity to slow relaxation processes.

Spatial harmonics of atomic populations (gratings) can be produced by the nonlinear interaction of counterpropagating radiation fields with the atoms. The time development of the spatial harmonics is extremely sensitive to velocity-changing processes such as collisions, atomic recoil on the absorption or emission of radiation and acceleration in an external field. An effective means for probing the ground state gratings is the so-called Grating-Stimulated Echo (GSE) [1]. The physical mechanisms that gives rise to the GSE signal is the same or similar to those responsible for two-photon echoes [2], interference of matter waves [3-6], atomic interferometry [7-9] and echoes in standing wave fields [10-15].

A complete theory of the GSE requires one to take into account the internal structure of the levels, including magnetic state degeneracy and fine or hyperfine splitting. In addition to ground state population gratings, magnetic alignment or orientation ground state gratings can be induced when polarized counterpropagating waves drive transitions between different ground or excited state sublevels. The ground state gratings can be probed with a traveling wave pulse, resulting in coherent emission which can be distinguished from the probe by its direction of propagation and polarization [16]. In a previous article [16] two specific excitation-detection schemes were described. In the Magnetic-Grating Free-Induction Decay (MGFID) a pulse of counterpropagating waves produces a coherence among ground state magnetic sublevels having a spatial dependence that varies as $\exp ( \pm 2 i k z)$ where $\mathbf{k}=k \hat{\mathbf{z}}$ is the wave vector of one of the fields. Following some delay $t_{\mathrm{p}}$, a traveling wave pulse is used to probe the gratings. Since the grating decays on a time scale of order $(2 k u)^{-1}(u=$ most probable atomic speed) owing to Doppler dephasing, the MGFID can probe only those relaxation processes with $t \geqq(2 k u)^{-1}$. This time can be extended if one uses two pulses of counterpropagating waves, separated in time by $T[\mathrm{Mag}-$ netic-Grating Echo (MGE)]. These pulses lead to a dephasing-rephasing of the second harmonic of the ground state magnetic coherence which is subsequently probed by a traveling wave pulse at time $t \approx 2 T$. In this manner, the Doppler dephasing is effectively canceled. As a consequence the MGE can be used to probe ground state relaxation for time scales $T \gg(k u)^{-1}$. 
In [16] it was assumed that the excitation had a sufficiently long temporal width to insure that the optical coherence and excited state density matrix adiabatically followed the incident fields. The opposite limiting situation is the subject of this article. We consider MGFID and MGE excited by ultrafast pulses, having a temporal width that is smaller than all other relevant times in the problem, such as the inverse hyperfine splitting of the levels, $(2 k u)^{-1}$, excited state lifetime $\Gamma^{-1}$. Our discussion is limited to situations in which $\Gamma / k u \gg 1$, applicable for atoms cooled to or below the Doppler limit of laser cooling [17]. In this limit the coherently emitted signal induced by the probe pulse has a temporal width of order $\Gamma^{-1}$. The hyperfine splitting can be resolved after spectral decomposition of the signal if $\Gamma$ is smaller than this splitting. Thus, one has a direct method for measuring hyperfine splittings. In comparison with other coherent transient techniques [18], the MGFID and MGE benefit from the fact that the signals have a temporal width much larger than that of the probe pulse, propagate in a direction opposite to the probe and can have a polarization orthogonal to that of the probe.

In Sect. 1 we outline the calculations and give final expressions for the MGFID and MGE signals in a perturbation theory limit. Details of the calculations are presented in the Appendix. Section 2 is devoted to a discussion of the main features of the signals, including the results of a computer calculation applicable to the $\mathrm{D}_{2}$ line of $\mathrm{Na}$. These calculations serve as a basis for future planned calculations in which strong field effects and relaxation processes will be included.

\section{MGFID and MGE signals}

We consider the interaction of an ensemble of atoms with one or more radiation pulses. The pulse centered at $t=t_{i}$ has an electric field which can be written as

$E=\sum_{q}(-1)^{q} E_{-q} \hat{\varepsilon}_{q}+$ c.c.,

where

$E_{q}=\frac{1}{2} \psi\left(t-t_{i}\right) \sum_{j=1,2} \mathscr{E}_{j} \varepsilon_{q}^{(j)} \exp \left(i \mathbf{k}_{j} \cdot \mathbf{r}-\mathrm{i} \Omega_{j} t\right)$

and $\psi(t)$ is a pulse envelope function centered at $t=0$ having a temporal width $\tau$. The quantities $\mathscr{E}_{j}, \Omega_{j}, \mathbf{k}_{j}, \varepsilon_{q}^{(j)}$ are the amplitude, frequency, wave vector and spherical component of the polarization vector of field $j$, and $\ddot{\varepsilon}_{q}$ is a unit polarization vector, defined as

$\hat{\boldsymbol{\varepsilon}}_{ \pm q}=\mp(\hat{\mathbf{x}} \pm \mathrm{i} \hat{\mathbf{y}}) / \sqrt{2}, \quad \boldsymbol{\varepsilon}_{0}=\hat{\mathbf{z}}$.

It is seen from (1) that each pulse actually consists of a superposition of two pulses incident from different directions. The pulses interact with atoms having nuclear spin I and whose ground and excited states consist of manifolds of fine and hyperfine levels. The total electronic angular momenta of the ground and excited states are
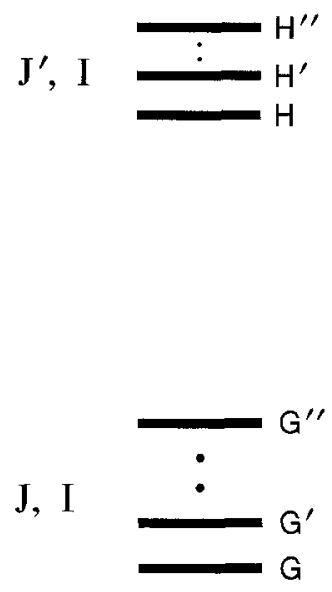

Fig. 1. Atomic energy level diagram

denoted by $J$ and $J^{\prime}$, respectively, while the total angular momenta associated with ground and excited state hyperfine levels are denoted by $G, G^{\prime}$, etc. and by $H, H^{\prime}$, etc., respectively (Fig. 1).

The pulse duration $\tau$ is assumed to satisfy

$$
\begin{aligned}
\tau \ll & \min \left(\left|\omega_{G G^{\prime}}^{-1},\right| \omega_{H H^{\prime}}^{-1}, \Gamma^{-1},(k u)^{-1},\left|\Omega_{j}-\omega_{H G}\right|^{-1},\right. \\
& \left.\left|\Omega_{j}-\Omega_{j^{\prime}}\right|^{-1}\right),
\end{aligned}
$$

where $\omega_{F F},=\left(E_{F}-E_{F}\right) / \hbar, E_{F}$ is the energy of state $F, \Gamma$ is the rate of spontaneous decay of the upper level, and $u$ is the most probable atomic speed. This inequality permits one to neglect the decay, hyperfine splitting and atomic motion during the pulses and consider the field to drive a transition between the two levels having angular momenta $J$ and $J^{\prime}$. On the other hand, one must account for decay, hyperfine splitting and atomic motion in the field-free intervals between the pulses. Using a representation of the density matrix in which electronic momenta and nuclear spin are decoupled to evaluate the atomic response during the pulses and one in which they are coupled to evaluate the response in the field-free intervals greatly simplifies the calculations (see Appendix).

Consider the interaction of the atoms with the pulse centered at $t_{1}=0$, consisting of two waves counterpropagating along the $z$ axis $\left(\mathbf{k}_{i}=k_{i} \hat{\mathbf{z}}, k_{1}=-k_{2}=k\right)$. (The more general case of arbitrary $\mathbf{k}_{1}$ and $\mathbf{k}_{2}$ is treated in the Appendix.) After the pulse, the excited state decays owing to spontaneous emission. Following this decay, the ground state density matrix $\varrho$ can still retain a memory of the atom-field interaction, including a spatial modulation induced by the counterpropagating waves. In the limit of weak excitation fields, one finds that the ground state density matrix contains a modulated component which, in the atomic rest frame, varies as

$\varrho(z, t) \propto \exp \left(-2 \mathrm{i} k z_{0}\right)$,

where $v$ is $z$-projection of the atomic velocity and $z_{0}=z-v t$ is the $z$-coordinate at the time of the interaction with the pulse. The $v$-dependence of the harmonic (4) implies that the ground state density matrix can be used to monitor velocity changes that occur on a time 
scale given by some effective ground state lifetime. For the spatially modulated term (4), the time scale is set by $(k u)^{-1}$, the inverse of the inhomogeneous width associated with the atomic velocity distribution. Even for atoms cooled below the Doppler limit, such that

$k u \ll \Gamma$,

the coherence time $(k u)^{-1}$ can be much smaller than some intrinsic ground state lifetime determined, for example, by the time the atom spends in the interaction volume. Thus, it is necessary to eliminate effects related to inhomogeneous broadening if the ultimate sensitivity to small velocity changes is to be obtained.

The echo technique allows one to achieve this goal. Following a two-quantum interaction with the first pulse, centered at $t_{1}=0$, and a four-quantum interaction with the second pulse, centered at $t_{2}=T$, the ground state density matrix has components varying as

$\varrho \propto \exp \left\{\mathrm{i}\left(k_{j_{1} j_{2}}\right)(z-v t)+\mathrm{i}\left(k_{j_{3} j_{4}}+k_{j_{j} j_{6}}\right)[z-v(t-T)]\right\}$,

where $j_{\mathrm{i}}=1,2(i=1-6), k_{11}=k_{22}=0, k_{12}=-k_{21}=2 k$. The term involving $k_{j_{1} j_{2}}$ is associated with the first pulse and that involving $k_{j_{3} j_{4}}+k_{j_{5} j_{6}}$ with the second. To suppress the limitation on observation times imposed by inhomogeneous atomic velocity distribution it is necessary that the total Doppler phase vanishes, i.e.,

$k_{j_{1} j_{2}} t+\left(k_{j_{3} j_{4}}+k_{j_{5} j_{6}}\right)(t-T)=0$.

For reasons, discussed below, related to probing the ground state distribution, we also require that (6) corresponds to a spatial harmonic varying as $\exp ( \pm 2 \mathrm{i} k z)$. This requirement leads to the condition

$k_{j_{1} j_{2}}+k_{j_{3} j_{4}}+k_{j_{5} j_{6}}=\mp 2 k$.

To satisfy both (7) and (8) for times $t>T$, one must have

$k_{j_{1} j_{2}}=-k_{j_{3} j_{4}}=-k_{j_{5} j_{6}}= \pm 2 k$.

For either sign, the echo occurs at

$t=2 T$.

In what follows, it is important to underline the fact that this echo occurs only if the "wave vector" change during the interaction with the second pulse satisfies

$k_{j_{3} j_{4}}+k_{j_{5} j_{6}}=\mp 4 k$.

The ground state density matrix elements (4) and (6) can be monitored by backscattering a probe pulse off the sample. The probe pulse is a traveling wave having electric field vector

$$
\begin{aligned}
\mathbf{E}_{\mathrm{p}}(\mathbf{r}, t)= & \frac{1}{2}(-1)^{q} \mathscr{E}_{\mathrm{p}} \varepsilon_{-q}^{(p)} \hat{\varepsilon}_{q} \\
& \times \psi\left(t-t_{\mathrm{p}}\right) \exp \left(-\mathrm{i} \Omega_{\mathrm{p}} t+\mathrm{i} \mathbf{k}_{\mathrm{p}} \cdot \mathbf{r}\right)+\text { c.c. },
\end{aligned}
$$

frequency $\Omega_{\mathrm{p}}$, wave vector $\mathbf{k}_{\mathrm{p}}$, amplitude $\mathscr{E}_{\mathrm{p}}$, polarization components $\varepsilon_{q}^{(p)}$ and envelope function $\psi_{\mathrm{p}}$. If this pulse is centered at a time $t_{\mathrm{p}}$ where the velocity-averaged second harmonics (4) or (6) are nonvanishing, and if the pulse propagates along the $z$-axis $\left(\mathbf{k}_{\mathrm{p}}=k \hat{\mathbf{z}}\right)$ then it induces a polarization wave, propagating in the $-\hat{\mathbf{z}}$ direction. General expressions are derived in the Appendix for a quantity $\mathscr{P}_{\text {s }}$ defined by

$\mathscr{P}_{\mathrm{s}}=\mathscr{P} \cdot \boldsymbol{\varepsilon}_{\mathrm{s}}^{*}$,

where $\mathscr{P}$ is the positive frequency component of the polarization and $\boldsymbol{\varepsilon}_{\mathrm{s}}$ is a complex unit vector. The quantity $\mathscr{P}_{\mathrm{s}}$ is the component of $\mathscr{P}$ in the direction $\boldsymbol{\varepsilon}_{\mathrm{s}}$ that we choose to measure the signal's polarization. The signal resulting from the backscattering of the probe is proportional to $\left|\mathscr{P}_{\mathrm{s}}\right|^{2}$, but we will simply refer to $\mathscr{P}_{\mathrm{s}}$ as "the signal".

If the atoms are in equilibrium before the action of the pulses, one obtains an MGFID signal

$$
\begin{aligned}
\mathscr{P}_{\mathrm{s}}= & \mathrm{i} N \mathscr{E}_{\mathrm{p}}\left|p_{J^{\prime} J}\right|^{2} \chi_{J^{\prime}}^{(2)}\left[\chi_{J^{\prime} J}^{(1)}\right]^{*} \tau_{\mathrm{p}} \tau^{2} \\
& \times \exp \left[-\Gamma / 2\left(t-t_{\mathrm{p}}\right)-\mathrm{i} k z-\mathrm{i} \Omega_{\mathrm{p}} t_{\mathrm{p}}\right]\left\langle\exp \left[\mathrm{i} k v\left(t+t_{\mathrm{p}}\right)\right]\right\rangle \\
& \times P_{H G}\left(t_{\mathrm{p}}\right) \exp \left[-\mathrm{i} \omega_{H G}\left(t-t_{\mathrm{p}}\right)\right],
\end{aligned}
$$

where

$P_{H G}\left(t_{\mathrm{p}}\right)=P_{H G}^{\mathrm{f}} \sum_{n} P_{H G}^{(n)} \exp \left(\mathrm{i} n B t_{\mathrm{p}} / 2\right)$,

$P_{H G}^{(n)}=\tilde{P}_{H G}^{(n)} / P_{H G}^{\mathrm{r}}$,

$P_{H G}^{\mathrm{f}}=\left(\sum_{n}\left|\widetilde{P}_{H G}^{(n)}\right|^{2}\right)^{1 / 2}$,

$$
\widetilde{P}_{H G}^{(n)}=(-1)^{H+G^{\prime}+1} 3 / 2 \delta_{n, \tilde{\omega}\left(G, G^{\prime}\right)} \lambda_{0}^{0}(K, p, s ; K, 2,1)
$$$$
\times\left\{K, H^{2}, G^{2}, G^{\prime 2}, J^{-2}, I^{-2}\right\}
$$$$
\times\left\{\begin{array}{ccc}
1 & 1 & K \\
G & G^{\prime} & H
\end{array}\right\}\left\{\begin{array}{lll}
J^{\prime} & H & I \\
G & J & 1
\end{array}\right\}\left\{\begin{array}{lll}
J^{\prime} & H & I \\
G^{\prime} & J & 1
\end{array}\right\} L_{K}\left(G, G^{\prime}\right)
$$

$$
\begin{aligned}
L_{K}\left(G, G^{\prime}\right)= & (-1)^{J^{\prime}-G-I}\left\{\begin{array}{ccc}
G & G^{\prime} & K \\
J & J & I
\end{array}\right\}\left\{\begin{array}{lll}
1 & 1 & K \\
J & J & J^{\prime}
\end{array}\right\} \\
& +(-1)^{J+G-1}\left[1-\mathrm{i}\left(\omega_{G^{\prime}}-\omega_{H^{\prime}} H^{\prime \prime}\right) / \Gamma\right]^{-1} \\
& \times\left\{J^{\prime 2}, H^{\prime 2}, H^{\prime \prime 2}\right\}\left\{\begin{array}{ccc}
J & G^{\prime} & I \\
H^{\prime} & J^{\prime} & 1
\end{array}\right\}\left\{\begin{array}{ccc}
J & G & I \\
H^{\prime \prime} & J^{\prime} & 1
\end{array}\right\} \\
& \times\left\{\begin{array}{ccc}
H^{\prime} & H^{\prime \prime} & K \\
G & G^{\prime} & 1
\end{array}\right\}\left\{\begin{array}{ccc}
H^{\prime} & H^{\prime \prime} & K \\
J^{\prime} & J^{\prime} & I
\end{array}\right\}\left\{\begin{array}{ccc}
1 & 1 & K \\
J^{\prime} & J^{\prime} & J
\end{array}\right\},(14+)
\end{aligned}
$$

and an MGE-signal

$$
\begin{aligned}
\mathscr{P}_{\mathrm{s}}= & \mathrm{i} \mathscr{E}_{\mathrm{p}}\left|\mathrm{p}_{J^{\prime} J} \chi_{J^{\prime} J}^{(1)} \chi_{J^{\prime} J}^{(2)}\right|^{2} \chi_{J^{\prime} J}^{(2)}\left[\chi_{J^{\prime} J}^{(1)}\right]^{*} \tau_{\mathrm{p}} \tau^{6} \\
& \times \exp \left[-\Gamma / 2\left(t-t_{\mathrm{p}}\right)-\mathrm{i} k z-\mathrm{i} \Omega_{\mathrm{p}} t_{\mathrm{p}}\right] \\
& \times\left\langle\exp \left[\mathrm{i} k v\left(t+t_{\mathrm{p}}-4 T\right)\right]\right\rangle P_{H G}\left(t_{\mathrm{p}}, T\right) \\
& \times \exp \left[-\mathrm{i} \omega_{H G}\left(t-t_{\mathrm{p}}\right)\right],
\end{aligned}
$$

where

$P_{H G}\left(t_{\mathrm{p}}, T\right)=P_{H G}^{\mathrm{e}} \sum_{n, m} P_{H G}^{(n, m)} \exp \left[\mathrm{i} \frac{B}{2}\left(n T+m t_{\mathrm{p}}\right)\right]$, 


$$
\begin{aligned}
P_{H G}^{(n, m)}= & \tilde{P}_{H G}^{(n, m)} \mid P_{H G}^{\mathrm{e}}, \\
P_{H G}^{\mathrm{e}}= & \left(\sum_{n, m}\left|\widetilde{P}_{H G}^{(n, m)}\right|^{2}\right)^{1 / 2} \\
\tilde{P}_{H G}^{(n, m)}= & -\frac{1}{16}(-1)^{H+G^{\prime}+K_{1}+Q_{c}} \delta_{n, \tilde{\omega}\left(G, G^{\prime}\right)-\tilde{\omega}\left(G_{1}, G_{1}^{\prime}\right)} \delta_{m, \tilde{\omega}\left(G, G^{\prime}\right)} \\
& \times\left\{K_{c}^{-1}, J^{-2}, I^{-2}, H^{2}, G, G^{\prime}, G_{1}, G_{1}^{\prime}\right\} \\
& \times \lambda_{Q_{c}}^{K_{c}}\left(\bar{K}, 2,1 ; \bar{K}_{1}, 2,1\right) \lambda_{-Q_{c}}^{K_{\mathrm{c}}}\left(K, p, s ; K_{1}, 1,2\right) \\
& \times\left\{\begin{array}{ccc}
1 & 1 & K \\
G & G^{\prime} & H
\end{array}\right\}\left\{\begin{array}{lll}
J^{\prime} & H & I \\
G & J & 1
\end{array}\right\}\left\{\begin{array}{lll}
J^{\prime} & H & I \\
G^{\prime} & J & 1
\end{array}\right\} \\
& \times \widetilde{D}_{\bar{K}_{c} \bar{K}_{1}}^{K K_{1}}\left(G^{\prime}, G ; G_{1}, G_{1}^{\prime}\right) L_{K_{1}}\left(G_{1}^{\prime}, G_{1}\right) .
\end{aligned}
$$

There is a summation convention implicit in these and subsequent equations in which repeated indices and symbols appearing on the right-hand side (rhs) of an equation are to be summed over, except if they also appear on the left-hand side (lhs) of the equation.

All quantities in these formulas are defined in the Appendix. We refer to the quantities $P_{H G}^{\mathrm{f}}$ and $P_{H G}^{\mathrm{e}}$ as the average MGFID polarization and MGE polarization, respectively, for the $H \rightarrow G$ transition. The quantity $B$ is the ground state hyperfine splitting constant and the sums in (14b) and (15b) are consistent with the delta functions in (14e) and (15e). From (14b) and (15b), one sees that the MGFID and MGE signals can be expressed as Fourier expansions. The MGFID signal oscillates as $\exp \left(\mathrm{i} n B t_{\mathrm{p}} / 2\right)$ and the MGE signal as $\exp \left[\mathrm{i} B\left(n T+m t_{\mathrm{p}}\right) / 2\right]$, with amplitudes denoted by $\widetilde{P}_{H G}^{(n)}$ and $\tilde{P}_{H G}^{(n, m)}$, respectively.

\section{Discussion}

As was mentioned before, the MGFID and MGE signals have a duration of order $\Gamma^{-1}$. Each term in the sum over $G$ and $H$ in (14a) and (15a) corresponds to radiation on the transition $H \rightarrow G$. The hyperfine structure can be resolved if $\Gamma$ is smaller than the hyperfine splittings.

The radiation intensity for a given component is determined by the absolute square of the quantities in the lhs of (14b) and (15b). They are oscillatory functions of the delay between pulses $T$ and the time $t_{\mathrm{p}}$, having a characteristic period related to the inverse ground state hyperfine splitting. Consider, for example, the MGFID. The first pulse, having duration smaller than inverse hyperfine splitting, induces both diagonal and off-diagonal ground state density matrix elements, $\varrho(G, G)$ and $\varrho\left(G, G^{\prime}\right)$. Following the excitation pulse, the coherence $\varrho\left(G, G^{\prime}\right)$ oscillates at frequency $\omega_{G G^{\prime}}$, acquiring a phase $\varphi=\omega_{G G^{\prime}} t_{\mathrm{p}}$ at the time $t_{\mathrm{p}}$ at which the probe pulse acts. One can neglect any transient response during the temporally narrow probe pulse. As a result, the phase $\varphi$ is transferred from the ground state density matrix $\varrho\left(G, G^{\prime}\right)$ to the optical coherence $\varrho(G, H)$, which leads to quantum beats in the signal at frequency $\omega_{G G^{\prime}}$.

The final expressions (14) and (15) are evaluated numerically for arbitrary values of nuclear spin, electron- (a)

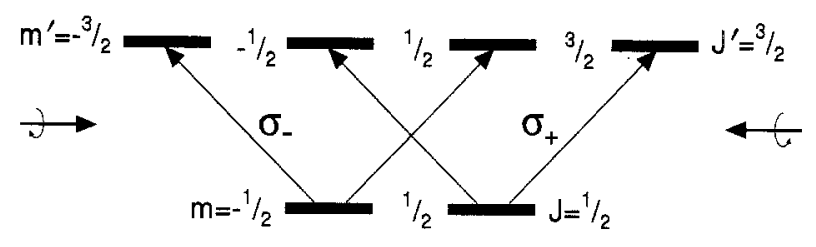

(b)

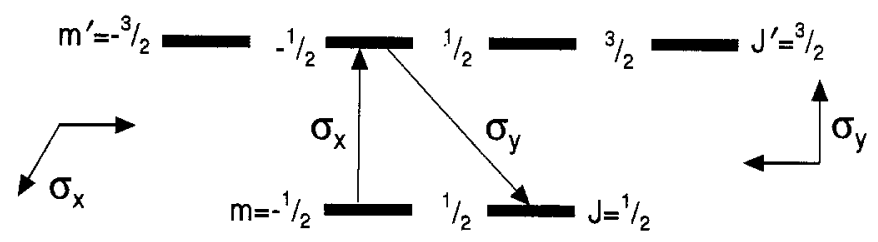

Fig. 2. Diagrams showing the coupling of the levels by the fields for a $J=1 / 2 \rightarrow J^{\prime}=3 / 2$ transition. To create a modulated ground state orientation, ground state sublevels must be coupled by the action of both fields; (a) $\sigma^{+}-\sigma^{-}$polarization with quantization axis along $\hat{\mathbf{z}}$ - there is no coupling of the $\pm 1 / 2$ ground state sublevels; (b) lin $\perp$ lin polarization with quantization axis along $\mathbf{x}-$ the $m=1 / 2$ ground state sublevel is connected to $m=-1 / 2$ with an amplitude proportional to $\mathrm{e}^{2 \mathrm{i} k z}$

ic angular momenta, hyperfine intervals and field polarizations. We present results for the specific case of the $\mathrm{D}_{2}$ line in $\mathrm{Na}$, where $J=1 / 2, \quad J^{\prime}=3 / 2 ; \quad I=3 / 2 ; \quad G=1,2$; $\omega_{G=2, G^{\prime}=1} / 2 \pi=1770 \mathrm{MHz} ; H=0,1,2,3 ; \omega_{H=1, H^{\prime}=0} / 2 \pi=$ $16 \mathrm{MHz} ; \omega_{H=2, H^{\prime}=1} / 2 \pi=34 \mathrm{MHz} ; \omega_{H=3, H^{\prime}=2} / 2 \pi=60 \mathrm{MHz}$ and the decay rate $\Gamma / 2 \pi=10 \mathrm{MHz}$.

Since the hyperfine interaction is frozen on the time scale of the ultrafast pulses, the results for $\mathrm{Na}$ can be expected to be similar to those for other alkali elements having the same electronic angular momenta of the ground and excited states. Consider, qualitatively, the interaction of an ultrafast pulse of counterpropagating waves on the $J=1 / 2 \rightarrow J^{\prime}=3 / 2$ transition. Two particular cases are shown in Fig. $2, \sigma^{+}-\sigma^{-}$and lin $\perp$ lin configurations. The $\sigma^{+}-\sigma^{-}$excitation pulse leads to a spatially modulated alignment $\varrho_{ \pm 2}^{2}\left(\varrho_{Q}^{K}\right.$ are components of the density matrix in an irreducible tensor basis), indicating a coherence between magnetic sublevels having $\Delta m= \pm 2$. For a $J=1 / 2$ to $J^{\prime}=3 / 2$ transition, alignment can be produced in the excited state only. Following the excitation pulse, one must recouple the electronic and nuclear angular momenta. The alignment in the $J^{\prime}=3 / 2$ states then translates into an alignment of the various excited state hyperfine levels. This alignment can then be transferred to the ground state hyperfine levels via spontaneous emission. The backscattering of the probe field from this ground state alignment grating leads to the MGFID signal.

The $\sigma^{+}-\sigma^{-}$excitation scheme does not lead to a MGE in Na. This result can be understood if one recalls that the second excitation pulse at $t=T$ must change the "wave vector" of the grating by $\pm 4 k(11)$. To obtain this change in the density matrix, one needs a change of $\pm 2 k$ in the state amplitudes. It is evident from Fig. 2a that for an arbitrary mixture of the ground state magnetic sub- 
levels, such a change cannot occur. Thus, the absence of two-quantum driving of the ground state by counterpropagating waves leads to the absence of the MGE on the transition $J=1 / 2 \rightarrow J^{\prime}=3 / 2$ for $\sigma^{+}-\sigma^{-}$pulse configurations. Two-quantum driving becomes possible in the case of lin $\perp$ lin pulse configuration (Fig. 2b) and it results in a MGE signal.

It might be noted that an amplitude picture is useful for a quantitative solution to the problem, since there is no relaxation during the atom field interaction. The amplitude equations can be significantly simpler than the corresponding density matrix equations, particularly in the case of strong pulses where the perturbative theory limit is no longer applicable.

The spatially modulated hyperfine coherence $\varrho\left(G, G^{\prime}\right)$ $\left(G \neq G^{\prime}\right)$ can be produced "directly" during the excitation pulse via two-quantum processes or "indirectly" following the pulse via spontaneous decay from the upper state. The contributions from spontaneous decay depend on the value of the energy denominator

$\left[1-\mathrm{i}\left(\omega_{G G^{\prime}}-\omega_{H H^{\prime}}\right) / \Gamma\right]^{-1}$

in (A18), which, in turn, reflects spontaneous decay processes of the type

$\varrho\left(H, H^{\prime}\right) \rightarrow \varrho\left(G, G^{\prime}\right)$.

If

$\left|\omega_{G G^{\prime}}\right| \gg \mid \omega_{H H^{\prime}}$

and

$\Gamma \ll\left|\omega_{G G^{\prime}}\right|$,

the element $\varrho\left(G, G^{\prime}\right)$ oscillates rapidly on the time scale of spontaneous emission. As a consequence one can replace expression (16) by $\delta_{G G^{\prime}}\left[1+\mathrm{i} \omega_{H H^{\prime}} / \Gamma\right]^{-1}$. In this limit spontaneous decay can lead to spatially modulated ground state coherence among magnetic sublevels of a given hyperfine state, but not to coherence between different ground state hyperfine levels. If, also,

$\Gamma \ll \omega_{H H^{\prime}}, H \neq H^{\prime}$,

then one can also neglect contributions from the spontaneous decay of the hyperfine off-diagonal elements of the excited state density matrix. The approximations (19, 20 ), which are approximately satisfied for $\mathrm{Na}$, simplify the numerical calculations.

As an example we calculate the MGFID and MGE signals exactly and using approximations $(19,20)$ for an initially unperturbed ground state and lin $\perp$ lin polarization of the pumping pulses $\left(\varepsilon^{(1)}=\hat{\mathbf{x}}, \boldsymbol{\varepsilon}^{(2)}=\hat{\mathbf{y}}\right)$. The probe is polarized along $\hat{\mathbf{x}}$, and component of the signal polarized in the $\hat{\mathbf{y}}$-direction is monitored.

The most intense emission is on the transition $G=2 \rightarrow H=3$, where the average polarizations associated with the MGFID and MGE signals are

$P_{32}^{\mathrm{f}}=0.72 \cdot 10^{-2}$

and

$P_{32}^{\mathrm{e}}=0.75 \cdot 10^{-4}$, respectively. The relative weights $W_{H G}^{\mathrm{s}}=\left|P_{H G}^{\mathrm{s}}\right|^{2}(\mathrm{~s}=\mathrm{f}$ or e) of the various hyperfine components are

$W_{32}^{\mathrm{f}}: W_{22}^{\mathrm{f}}: W_{21}^{\mathrm{f}}: W_{11}^{\mathrm{f}}: W_{01}^{\mathrm{f}}: W_{12}^{\mathrm{f}}=$

$1: 0.25: 0.18: 0.09: 0.02: 0.015$,

$W_{32}^{\mathrm{e}}: W_{12}^{\mathrm{e}}: W_{21}^{\mathrm{e}}: W_{22}^{\mathrm{e}}: W_{11}^{\mathrm{e}}: W_{01}^{\mathrm{e}}=$

$1: 0.46: 0.42: 0.35: 0.29: 0.13$.

Since $\left|\Gamma / \omega_{G G^{\prime}}\right| \approx 0.006$, these results differ by no more than $1 \%$ from those obtained using approximation (19). The corresponding results using approximation (20) are

$P_{32}^{\mathrm{f}}=0.73 \cdot 10^{-2}$,

$P_{32}^{\mathrm{e}}=0.75 \cdot 10^{-4}$,

$W_{32}^{\mathrm{f}}: W_{22}^{\mathrm{f}}: W_{21}^{\mathrm{f}}: W_{11}^{\mathrm{f}}: W_{01}^{\mathrm{f}}: W_{12}^{\mathrm{f}}=$

$1: 0.24: 0.21: 0.14: 0.04: 0.014$

$W_{32}^{\mathrm{e}}: W_{21}^{\mathrm{e}}: W_{12}^{\mathrm{e}}: W_{11}^{\mathrm{e}}: W_{22}^{\mathrm{e}}: W_{01}^{\mathrm{e}}=$

$1: 0.60: 0.54: 0.49: 0.36: 0.23$.

It is not surprising that approximation (20) yields results that differ from the exact ones, since values $\left|\Gamma / \omega_{H H^{\prime}}\right|$ as large as 0.6 occur in the $J^{\prime}=3 / 2$ manifold in $\mathrm{Na}$.
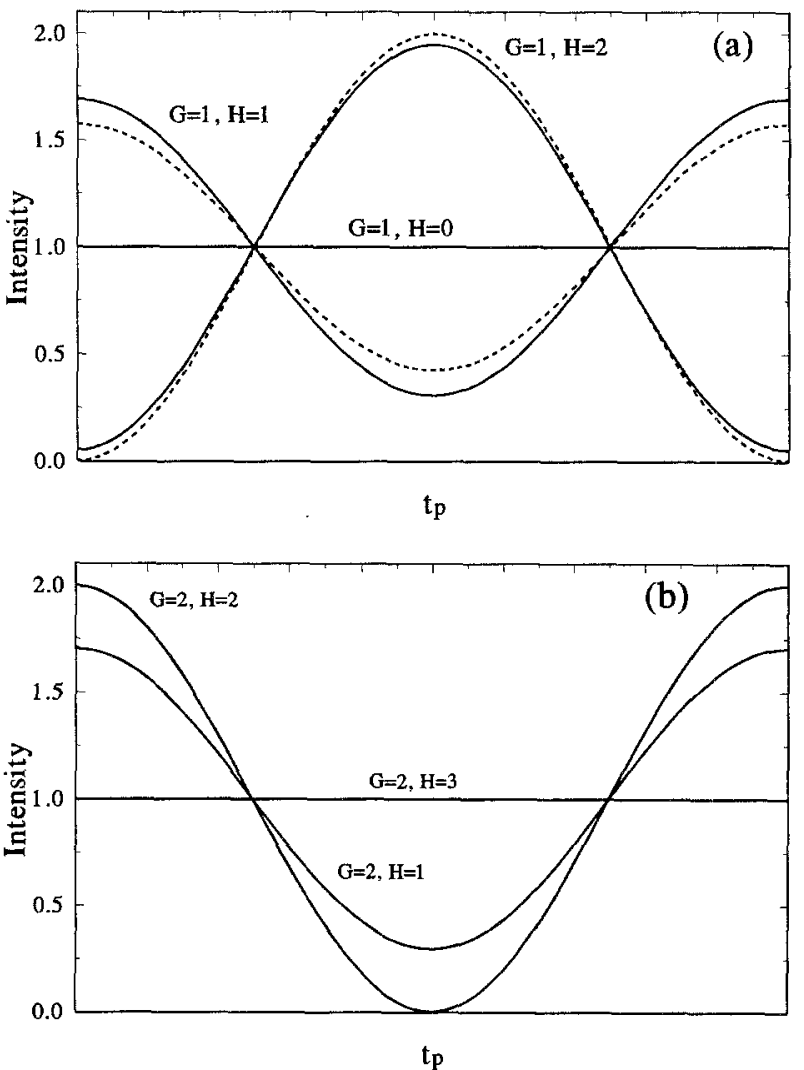

Fig. 3. a One period of the dependence of the MGFID spectral components terminating on the $G=1$ ground hyperfine state as a function of the delay $t_{\mathrm{p}}$ between probe and pump pulses. The intensity is in units of the average intensity for each curve. Dashed curves are plotted in approximation (20). The entire length of the $t_{\mathrm{p}}$ axis is $\pi / B$, where $B$ is the ground state hyperfine splitting constant. b The same for the components terminating on the $G=2$ ground hyperfine state 


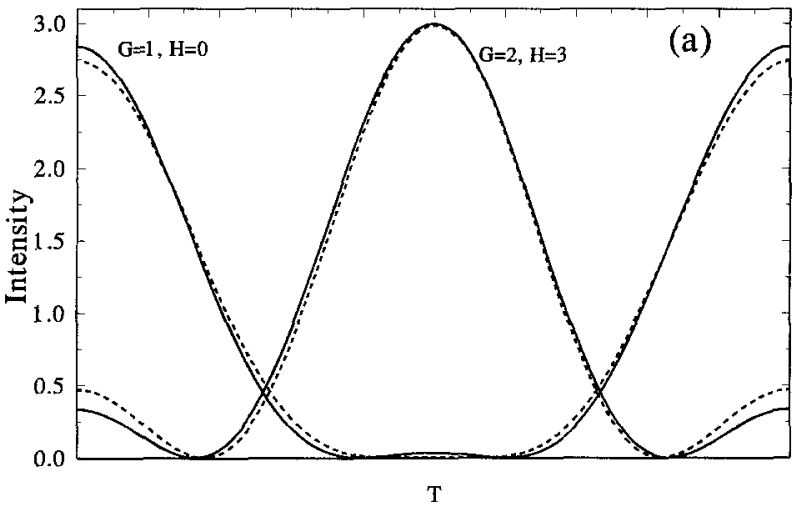

(c)

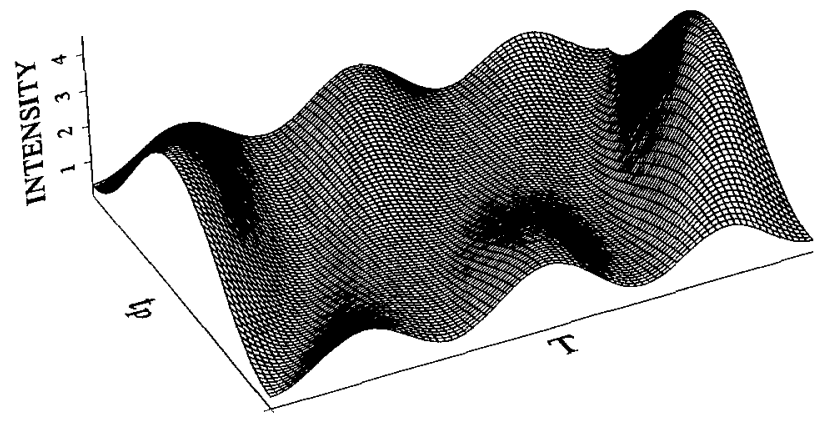

(e)

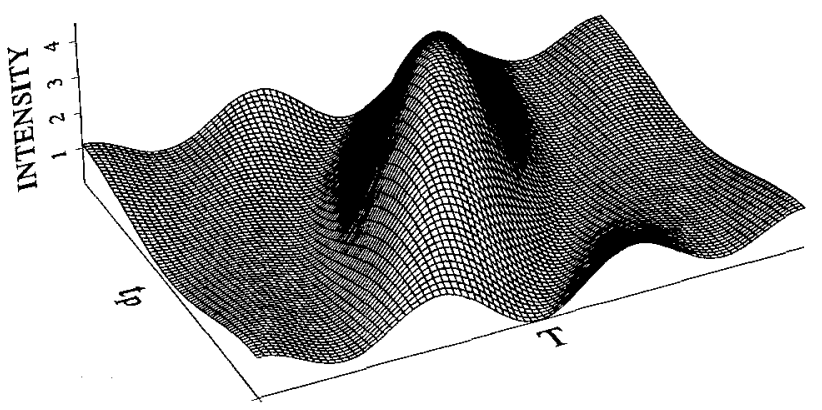

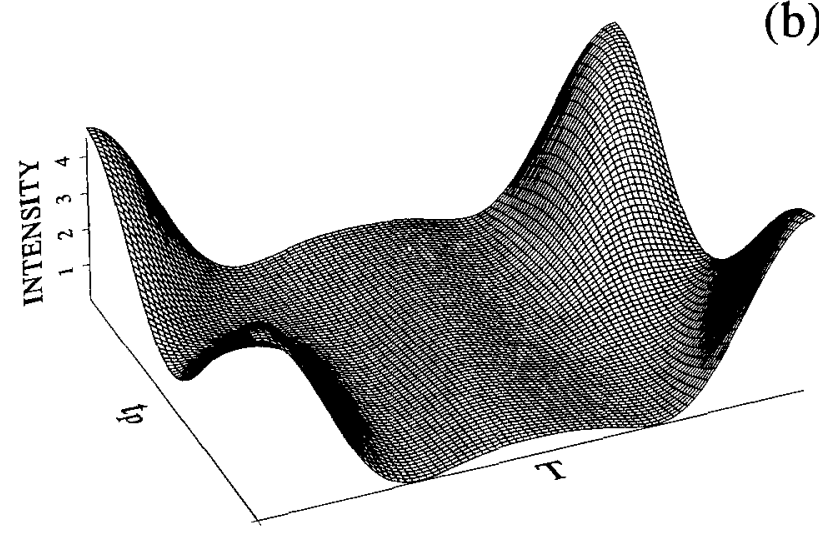

(d)

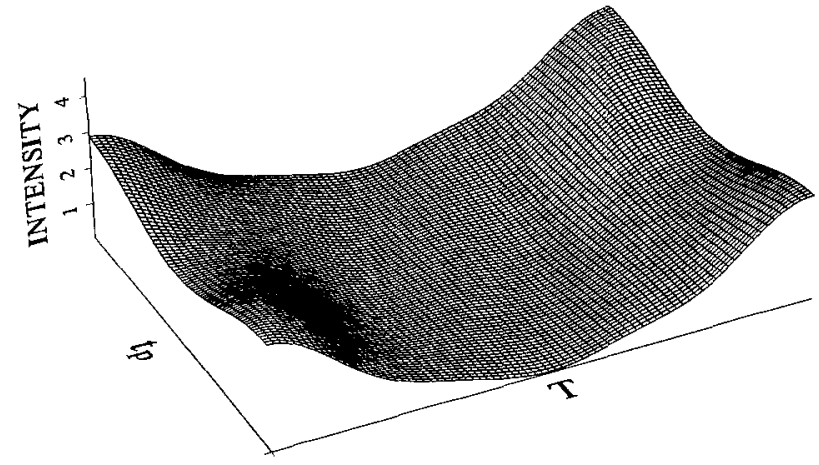

Fig 4. a Dependence of the MGE signal on delay $T$ between pumping pulses for the spectral components $G=1 \rightarrow H=0$ and $G=2 \rightarrow H=3$. The entire length of the $T$ axis is $\pi / B$ and the signal is independent of $t_{\mathrm{p}} . \mathbf{b}-\mathbf{e}$ Dependence of the MGE signal on the delays $T$ and $t_{\mathrm{p}}$. The axis length is $\pi / B$. b component $G=1 \rightarrow H=1$; c component $G=1 \rightarrow H=2$; d component $G=2 \rightarrow H=1$; e component $G=2 \rightarrow H=2$
Table 1. Nonvanishing values of the Fourier-harmonics in the MGFID signal decomposition (14b); in each position of the table, the first line corresponds to the exact calculations and second to the calculations using approximation (20)

\begin{tabular}{llll}
\hline Transition & $\overline{P_{H G}^{(-4)}}$ & $P_{H G}^{(0)}$ & $P_{H G}^{(4)}$ \\
\hline$G=1 \rightarrow H=0$ & & 1 & \\
$G=1 \rightarrow H=1$ & $-0.373+\mathrm{i} 0.004$ & -0.928 & \\
& -0.301 & -0.954 & \\
$G=1 \rightarrow H=2$ & $-0.813+\mathrm{i} 0.008$ & 0.582 & \\
& -0.735 & 0.688 & $-0.925-\mathrm{i} 0.009$ \\
$G=2 \rightarrow H=1$ & & -0.381 & -0.924 \\
& & -0.382 & $-0.688-\mathrm{i} 0.006$ \\
$G=2 \rightarrow H=2$ & & -0.725 & -0.677 \\
$G=2 \rightarrow H=3$ & -0.736 & \\
\hline
\end{tabular}


Table 2. The same as in Table 1 for Fourier harmonics in the decomposition (15b) of the MGE signal

\begin{tabular}{|c|c|c|c|c|c|c|c|c|c|}
\hline$G \rightarrow H$ & $P_{H G}^{(-8,4)}$ & $P_{H G}^{(-4,0)}$ & $P_{H G}^{(-4,4)}$ & $\left.P_{H G}^{(0,}-4\right)$ & $P_{H G}^{(0,0)}$ & $P_{H G}^{(0,4)}$ & $P_{H G}^{(4,-4)}$ & $P_{H G}^{(4,0)}$ & $P_{H G}^{(8,-4)}$ \\
\hline $1 \rightarrow 0$ & & $\begin{array}{c}-0.468+ \\
\mathrm{i} 0.005 \\
-0.431\end{array}$ & & & $\begin{array}{l}-0.750 \\
-0.7922\end{array}$ & & & $\begin{array}{c}-0.468- \\
\mathrm{i} 0.005 \\
-0.431\end{array}$ & \\
\hline $1 \rightarrow 1$ & & $\begin{array}{c}-0.575+ \\
\mathrm{i} 0.006 \\
-0.536\end{array}$ & & $\begin{array}{c}-0.057+ \\
i 0.002 \\
-0.045\end{array}$ & $\begin{array}{l}-0.432 \\
-0.563\end{array}$ & & $\begin{array}{c}-0.282+ \\
\mathrm{i} 0.001 \\
-0.252\end{array}$ & $\begin{array}{c}-0.575+ \\
i 0.006 \\
-0.536\end{array}$ & $\begin{array}{c}-0.265- \\
\mathrm{i} 0.001 \\
-0.208\end{array}$ \\
\hline $1 \rightarrow 2$ & & $\begin{array}{r}0.418- \\
\mathrm{i} 0.004\end{array}$ & & $\begin{array}{c}-0.142+ \\
\mathrm{i} 0.005\end{array}$ & $\begin{array}{r}-0.503 \\
0.426\end{array}$ & & $\begin{array}{c}0.116- \\
\mathrm{i} 0.002\end{array}$ & $\begin{array}{c}-0.536 \\
0.418+ \\
\mathrm{i} 0.004\end{array}$ & $\begin{array}{c}-0.208 \\
-0.659- \\
\mathrm{i} 0.002\end{array}$ \\
\hline $2 \rightarrow 1$ & $-0.667+$ & $\begin{array}{c}0.425 \\
-0.115+\end{array}$ & $-0.711-$ & -0.121 & $\begin{array}{l}0.552 \\
0.057\end{array}$ & $-0.144-$ & 0.052 & $\begin{array}{r}0.425 \\
-0.115-\end{array}$ & -0.562 \\
\hline $2 \rightarrow 1$ & $\begin{array}{r}\mathrm{i} 0.002 \\
-0.622\end{array}$ & $\begin{array}{r}-0.115+ \\
10.001 \\
-0.109\end{array}$ & $\begin{array}{r}\mathrm{i} 0.002 \\
-0.756\end{array}$ & & 0.057 & $\begin{array}{c}-0.144- \\
\mathrm{i} 0.005 \\
-0.134\end{array}$ & & $\begin{array}{c}-0.115- \\
10.001 \\
-0.109\end{array}$ & \\
\hline $2 \rightarrow 2$ & $\begin{array}{c}-0.722+ \\
i 0.003\end{array}$ & $\begin{array}{c}-0.361+ \\
\mathrm{i} 0.003\end{array}$ & $\begin{array}{l}0.127+ \\
\mathrm{i} 0.002\end{array}$ & & 0.422 & $\begin{array}{c}-0.156- \\
\mathrm{i} 0.006\end{array}$ & & $\begin{array}{c}-0.361- \\
\mathrm{i} 0.003\end{array}$ & \\
\hline & -0.722 & -0.375 & 0.006 & & 0.412 & -0.156 & & -0.375 & \\
\hline $2 \rightarrow 3$ & & $\begin{array}{l}0.578- \\
i 0.006\end{array}$ & & & -0.576 & & & $\begin{array}{l}0.578+ \\
\mathrm{i} 0.006\end{array}$ & \\
\hline & & 0.604 & & & -0.520 & & & 0.604 & \\
\hline
\end{tabular}

The oscillating dependencies of the radiation on $t_{\mathrm{p}}$ for the MGFID and on $t_{\mathrm{p}}$ and $T$ for the MGE are shown in Figs. 3 and 4, respectively. The values of the Fourier harmonics (14c) and (15c), used for these plots, are presented in Tables 1 and 2 , respectively.

Condition (20) is necessary to resolve the hyperfine structure; if condition (20) is not satisfied, one has to consider the total signals. Even though we present results for the various hyperfine components, the total signals can be constructed from the values of the average polarizations (21), relative weights (22) and the harmonic amplitudes given in Tables 1 and 2 .

Using excitation schemes involving ultrafast pulses, we have shown how the MGFID and MGE signals can be used to obtain information on the various hyperfine splittings. The coherent signal that is generated contains spectral components at spacings corresponding to the various hyperfine transition frequencies. To eliminate effects related to Doppler broadening, we have considered only the limiting case of atoms cooled to or below the Doppler limit of laser cooling, for which $\Gamma \gg k u$. If $k u \gg \Gamma$, the various spectral components can be resolved only for hyperfine splittings greater than $2 k u$. On the other hand, even if $k u \gg \Gamma$, the MGE signal for $t=t_{\mathrm{p}}=2 T$ is essentially Doppler-free. As a consequence, one can monitor collisional shifts of ground state hyperfine transitions using the MGE technique.

Acknowledgements. This work is supported by the National Science Foundation through Grant No. PHY-9396245, by the U.S. Army Research Office and by the NSF Center for Ultrafast Optical Science.

\section{Appendix}

Two bases for an expansion of the density matrix multipoles are used below. The first, given by

$$
\begin{aligned}
\varrho_{Q}^{K}\left(F_{1}, F_{2}\right)= & (-1)^{F_{1}-m_{1}}\{K\}\left(\begin{array}{rrr}
F_{1} & F_{2} & K \\
m_{1} & -m_{2} & -Q
\end{array}\right) \\
& \times\left\langle F_{1}, m_{1} ! \varrho \mid F_{2}, m_{2}\right\rangle,
\end{aligned}
$$

where

$\left\{X_{1}^{\left(n_{1}\right)} \ldots X_{\mathrm{s}}^{\left(n_{s}\right)}\right\}=\left[\left(2 X_{1}+1\right)^{n_{1}} \ldots\left(2 X_{\mathrm{s}}+1\right)^{n_{s}}\right]^{1 / 2}$,

(:::) is a $3-J$ symbol [19], and $F_{1}$ and $F_{2}$ can be equal to the total angular momenta of the ground or excited states hyperfine sublevel $G$ or $H$, is convenient for describing the free evolution of the density matrix. The other, given by

$$
\begin{aligned}
\varrho_{Q_{e} Q_{n}}^{K_{o} K_{n}}\left(J_{1}, J_{2}\right)= & (-1)^{J_{1}-m_{1}+I-m_{n}}\left\{K_{e}, K_{n}\right\}\left(\begin{array}{rrr}
J_{1} & J_{2} & K_{e} \\
m_{1} & -m_{2} & -Q_{e}
\end{array}\right) \\
& \times\left(\begin{array}{ccc}
I & I & K_{n} \\
m_{n}-m_{n}^{\prime} & -Q_{n}
\end{array}\right) \\
& \times\left\langle J_{1}, m_{1} ; I, m_{n}|\varrho| J_{2}, m_{2} ; I, m_{n}^{\prime}\right\rangle, \quad \text { (A3) }
\end{aligned}
$$

where $J_{1}$ and $J_{2}$ can be equal to the electronic angular momenta of the ground or excited state $J$ or $J^{\prime}$, is useful in calculating the atomic response to an ultrafast pulse. The connection between the representations is given by

$$
\begin{aligned}
\varrho_{Q}^{K}\left(F_{1}, F_{2}\right)= & (-1)^{K_{e}+K_{n}+Q}\left\{K, K_{e}, K_{n}, F_{1}, F_{2}\right\} \\
& \times\left(\begin{array}{rrr}
K_{e} & K_{n} & K \\
Q_{e} & Q_{n} & -Q
\end{array}\right)\left\{\begin{array}{rrr}
K_{e} & K_{n} & K \\
J_{1} & I & F_{1} \\
J_{2} & I & F_{2}
\end{array}\right\} \\
& \times \varrho_{Q_{e}{ }_{e} K_{n}}^{K_{n}}\left(J_{1}, J_{2}\right),
\end{aligned}
$$




$$
\begin{aligned}
\varrho_{Q_{e} Q_{n}}^{K_{n} K_{n}}\left(J_{1}, J_{2}\right)= & (-1)^{K_{e}+K_{n}+Q}\left\{K, K_{e}, K_{n}, F_{1}, F_{2}\right\} \\
& \times\left(\begin{array}{llr}
K_{e} & K_{n} & K \\
Q_{e} & Q_{n} & -Q
\end{array}\right)\left\{\begin{array}{lll}
K_{e} & K_{n} & K \\
J_{1} & I & F_{1} \\
J_{2} & I & F_{2}
\end{array}\right\} \\
& \times \varrho_{Q}^{K}\left(F_{1},{ }^{\circ} F_{2}\right),
\end{aligned}
$$

where $\{: \vdots:\}$ is a 9-J symbol.

Consider first the interaction of an atom with the ultrafast pulse (1b). The density matrix equations have been given previously $[20,21]$. Neglecting hyperfine splitting and radiative processes during the pulse, one arrives at

$$
\begin{aligned}
\grave{\varrho}_{Q_{e} Q_{n}}^{K_{e} K_{n}}(J, J)= & \mathrm{i}\left\{\psi\left(t-t_{i}\right) \chi_{J^{\prime} J}^{(j)}(-1)^{2 J} \mathrm{e}^{\mathrm{i}\left(\mathbf{k}_{j} \cdot \mathbf{r}-\Omega_{j} t\right)}(-1)^{q}\right. \\
& \times \varepsilon_{-q}^{(j)} \varrho_{Q_{e}^{\prime} Q_{n}}^{K_{e}^{\prime} K_{n}}\left(J, J^{\prime}\right)-\left[\psi\left(t-t_{i}\right) \chi_{J^{\prime} J}^{(j)}\right]^{*} \\
& \times(-1)^{2 J^{\prime}+1+K_{e}^{\prime}+K_{e}+Q_{e}^{\prime}} \mathrm{e}^{-\mathrm{i}\left(\mathbf{k}_{j} \cdot \mathbf{r}-\Omega_{j} t\right)} \\
& \left.\times\left(\varepsilon_{q}^{(j)}\right)^{*}\left[\varrho_{-}^{K_{e}^{\prime} K_{n}}\left(J, Q_{e}^{\prime} Q_{n}\right)\right]^{*}\right\} \Lambda_{Q_{e}^{\prime} q Q_{e}}^{K_{e}^{\prime} 1 K_{e}}\left(J, J, J^{\prime}\right),
\end{aligned}
$$

$$
\begin{aligned}
& \dot{\varrho}_{Q_{e} Q_{n}}^{K_{e} K_{n}}\left(J^{\prime}, J^{\prime}\right)=-\mathrm{i}\left\{\psi\left(t-t_{i}\right) \chi_{J^{\prime} J}^{(j)}(-1)^{2 J^{\prime}+K_{e}^{\prime}+K_{e}+1+q} \mathrm{e}^{\mathrm{i}\left(\mathbf{k}_{j} \cdot \mathbf{r}-\Omega_{j} t\right)}\right. \\
& \times \varepsilon_{-q}^{(j)} \varrho_{Q_{e}^{\prime} Q_{n}}^{K_{e}^{\prime} K_{n}}\left(J, J^{\prime}\right)-\left[\psi\left(t-t_{i}\right) \chi_{J^{\prime} J}^{(j)}\right]^{*} \\
& \times(-1)^{2 J+Q_{e}^{\prime}} \mathrm{e}^{-\mathrm{i}\left(\mathbf{k}_{j} \cdot \mathbf{r}-\Omega_{j} t\right)} \\
& \left.\times\left(\varepsilon_{q}^{(j)}\right)^{*}\left[\varrho_{-Q_{e}^{\prime} Q_{n}}^{K_{e}^{\prime} K_{n}}\left(J, J^{\prime}\right)\right]^{*}\right\} \\
& \times A_{Q_{e}^{\prime} q Q_{e}}^{K_{e}^{\prime} 1 K_{0}}\left(J^{\prime}, J^{\prime}, J\right),
\end{aligned}
$$

$$
\begin{aligned}
\dot{\varrho}_{Q_{e}^{e} Q_{n}}^{K_{e} K_{n}}\left(J, J^{\prime}\right)= & -\mathrm{i} \omega_{J J^{\prime}} \varrho_{Q_{e} Q_{n}}^{K_{e} K_{n}}\left(J, J^{\prime}\right)+\mathrm{i}\left[\psi\left(t-t_{i}\right) \chi_{J^{\prime} J}^{(j)}\right]^{*} \\
& \times(-1)^{2 J} \mathrm{e}^{-\mathrm{i}\left(\mathrm{k}_{j} \cdot \mathbf{r}-\Omega_{j} t\right)}\left(\varepsilon_{q}^{(j)}\right)^{*}\left\{\Lambda_{Q_{e}^{\prime} q Q_{e}}^{K_{e}^{\prime} 1 K_{e}}\left(J^{\prime}, J, J\right)\right. \\
& \times Q_{Q_{e}^{\prime} Q_{n}^{\prime} K_{n}}^{K_{1}^{\prime}}(J, J)-(-1)^{Q_{e}^{\prime}+K_{e}^{\prime}-K_{e}+1} \\
& \left.\times A_{Q_{e}^{\prime} q Q_{e}}^{K_{e}^{\prime} 1 K_{e}}\left(J, J^{\prime}, J^{\prime}\right)\left[\varrho_{-Q_{e}^{\prime} Q_{n}}^{K_{e}^{\prime} K_{n}}\left(J^{\prime}, J^{\prime}\right)\right]^{*}\right\} \quad(\mathrm{A} 5 \mathrm{c})
\end{aligned}
$$

where

$\chi_{J^{\prime} J}^{(j)}=p_{J^{\prime} J} \mathscr{E}_{j} /(2 \sqrt{3} \hbar)$

is a Rabi frequency,

$$
\begin{aligned}
\Lambda_{Q^{\prime} q Q}^{K^{\prime} k K}(A, B, C)= & (-1)^{K+K^{\prime}+Q}\left\{k, K, K^{\prime}\right\} \\
& \times\left(\begin{array}{rrr}
K^{\prime} & k & K \\
Q^{\prime} & q & -Q
\end{array}\right)\left\{\begin{array}{lll}
K^{\prime} & k & K \\
A & B & C
\end{array}\right\},
\end{aligned}
$$

$\{:::\}$ is a $6-\mathrm{J}$ symbol and the time derivatives are total time derivatives in the sense that

$\frac{d}{d t}=\frac{\partial}{\partial t}+\mathbf{v} \cdot \frac{\partial}{\partial \mathbf{r}}$

where $\mathbf{v}$ is the atomic velocity.

One can express the coherence $\varrho_{Q_{e} Q_{n} K_{n}}^{K_{n}}\left(J, J^{\prime}\right)$ in terms of the excited and ground state density matrix elements to arrive at the system of integral equations

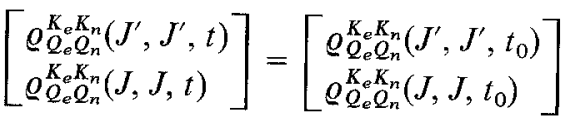

$$
\begin{aligned}
& +\exp \left(i \mathbf{k}_{j j^{\prime}} \cdot \mathbf{r}\right) \chi_{J^{\prime} J}^{(j)}\left[\chi_{J^{\prime} J}^{\left(j^{\prime}\right)}\right]^{*} \\
& \times(-1)^{Q^{\prime}} \varepsilon_{\bar{Q}}^{\bar{K}}\left(j, j^{\prime}\right)\left(\begin{array}{rrr}
K_{e} & K_{e}^{\prime} & \bar{K} \\
Q_{e} & -Q_{e}^{\prime} & \bar{Q}
\end{array}\right) \\
& \times\left[\begin{array}{ll}
C_{K_{e}^{\prime} \bar{K}}^{K_{e}}\left(J^{\prime}, J^{\prime}\right) & C_{K_{e}^{\prime} \bar{K}}^{K_{e}}\left(J^{\prime}, J\right) \\
C_{K_{e}^{e} \bar{K}}^{K_{e}}\left(J, J^{\prime}\right) & C_{K_{e}^{e} \bar{K}}^{K_{e}}(J, J)
\end{array}\right] \\
& \times \int_{t_{0}}^{t} d t^{\prime} \int_{t_{0}}^{t} d t^{\prime \prime} \psi\left(t^{\prime}-t_{i}\right) \psi^{*}\left(t^{\prime \prime}-t_{i}\right) \\
& \times\left[\begin{array}{l}
\varrho_{Q_{e}}^{K_{e} K_{n}}\left(U_{n}^{\prime}, J^{\prime}, t^{\prime \prime}\right) \\
\varrho_{Q_{e} Q_{Q}}^{K_{n}}\left(J, J, t^{\prime \prime}\right)
\end{array}\right],
\end{aligned}
$$

where

$\varepsilon_{Q}^{K}\left(j, j^{\prime}\right)=(-1)^{q}\{K\}\left(\begin{array}{rrr}1 & 1 & K \\ q & q^{\prime} & -Q\end{array}\right) \varepsilon_{q}^{(j)}\left[\varepsilon_{-q^{\prime}}^{\left(j^{\prime}\right)}\right]^{*}$

is a component of a coupled basis polarization tensor [22],

$$
\begin{aligned}
C_{K^{\prime} \bar{K}}^{K}\left(J^{\prime}, J^{\prime}\right)= & -3(-1)^{J-J^{\prime}}\left\{K, K^{\prime}, \bar{K}\right\}\left[1+(-1)^{K+K^{\prime}+\bar{K}}\right] \\
& \times\left\{\begin{array}{lll}
1 & 1 & \bar{K} \\
J^{\prime} & J^{\prime} & J
\end{array}\right\}\left\{\begin{array}{lll}
K & K^{\prime} & \bar{K} \\
J^{\prime} & J^{\prime} & J^{\prime}
\end{array}\right\},
\end{aligned}
$$

$$
\begin{aligned}
C_{K^{\prime}}^{K}\left(J^{\prime}, J\right)= & -6(-1)^{K^{\prime}}\left\{K, K^{\prime}, \bar{K}\right\}\left\{\begin{array}{lll}
K & K^{\prime} & \bar{K} \\
J^{\prime} & J & 1 \\
J^{\prime} & J & 1
\end{array}\right\}, \quad \text { (A11b) } \\
C_{K^{\prime}}^{K}\left(J, J^{\prime}\right)= & -6(-1)^{K}\left\{K, K^{\prime}, \bar{K}\right\}\left\{\begin{array}{lll}
K & K^{\prime} & \bar{K} \\
J & J^{\prime} & 1 \\
J & J^{\prime} & 1
\end{array}\right\}, \quad \text { (A11c) } \\
C_{\bar{K}^{\prime} \bar{K}}^{K}(J, J)= & -3(-1)^{J-J^{\prime}+\bar{K}}\left\{K, K^{\prime}, \bar{K}\right\}\left[1+(-1)^{K+K^{\prime}+\bar{K}}\right] \\
& \times\left\{\begin{array}{lll}
1 & 1 & \bar{K} \\
J & J & J^{\prime}
\end{array}\right\}\left\{\begin{array}{lll}
K & K^{\prime} & \bar{K} \\
J & J & J
\end{array}\right\},
\end{aligned}
$$

and conditions (3) have been invoked.

In general, for arbitrary quantum numbers of the atomic levels and field polarization, one can reach an analytical solution of (A9) in perturbation theory, when $\left|\chi_{J^{\prime} J}^{(j)}\right| \tau \ll 1$.

Solving (A9) to fourth order in the parameter (A12), and applying (A1), one finds a change in density matrix elements

$\Delta \varrho_{Q}^{K}\left(F, F^{\prime}\right)=\varrho_{Q}^{K}\left(F, F^{\prime} ;+\right)-\varrho_{Q}^{K}\left(F, F^{\prime} ;-\right)$,

where $\varrho_{Q}^{K}\left(F, F^{\prime} ; \pm\right)$ are the density matrix elements just after and before the field action, given by:

$\Delta \varrho_{Q}^{K}\left(F, F^{\prime}\right)=\Delta^{(2)} \varrho_{Q}^{K}\left(F, F^{\prime}\right)+\Delta^{(4)} \varrho_{Q}^{K}\left(F, F^{\prime}\right)$,

where 


$$
\begin{aligned}
& \Delta^{(2)} \varrho_{Q}^{K}\left(F_{1}, F_{1}^{\prime}\right)=(-1) Q^{Q^{\prime}} \chi_{J^{\prime} J}^{(j)}\left[\chi_{J^{\prime} J}^{\left(j^{\prime}\right)}\right]^{*} \frac{\tau^{2}}{2} \exp \left(\mathrm{i} \mathbf{k}_{j j^{\prime}} \cdot \mathbf{r}\right) \\
& \times\left(\begin{array}{rrr}
K & K^{\prime} & \bar{K} \\
Q & -Q^{\prime} & \bar{Q}
\end{array}\right) \varepsilon_{\bar{Q}}^{\bar{K}}\left(j, j^{\prime}\right) \\
& \times \bar{C}_{K^{\prime} \bar{K}}^{K}\left(F_{1}, F_{1}^{\prime} ; F_{2}, F_{2}^{\prime}\right) \\
& \times \varrho_{Q^{\prime}}^{K^{\prime}}\left(F_{2}, F_{2}^{\prime},-\right), \\
& \bar{C}_{K^{\prime} \bar{K}}^{K}\left(F_{1}, F_{1}^{\prime} ; F_{2}, F_{2}^{\prime}\right)=(-1)^{K+K^{\prime}+\vec{K}+K_{n}} \\
& \times\left\{K, K^{\prime}, K_{e}, K_{n}^{2}, K_{e}^{\prime}, F_{1}, F_{1}^{\prime}, F_{2}, F_{2}^{\prime}\right\} \\
& \times\left\{\begin{array}{lll}
K & K^{\prime} & \bar{K} \\
K_{\mathrm{e}}^{\prime} & K_{e} & K_{n}
\end{array}\right\} C_{K_{e}^{\prime} \bar{K}}^{K_{e}}\left(J_{1}, J_{2}\right) \\
& \times\left\{\begin{array}{lll}
K_{e} & K_{n} & K \\
J_{1} & I & F_{1} \\
J_{1} & I & F_{1}^{\prime}
\end{array}\right\} \\
& \times\left\{\begin{array}{lll}
K_{\mathrm{e}}^{\prime} & K_{n} & K^{\prime} \\
J_{2} & I & F_{2} \\
J_{2} & I & F_{2}^{\prime}
\end{array}\right\}
\end{aligned}
$$

$\Delta^{(4)} Q_{Q}^{K}\left(F_{1}, F_{1}^{\prime}\right)=(-1)^{Q^{\prime \prime}} \chi_{J^{\prime} J}^{(j)}\left[\chi_{J^{\prime} J}^{\left(j_{1}\right)}\right]^{*} \chi_{J^{\prime} J}^{\left(j^{\prime}\right)}\left[\chi_{J^{\prime} J}^{\left(j^{\prime}\right)}\right]^{*} \frac{\tau^{4}}{24}$

$$
\begin{aligned}
& \times \exp \left[\mathrm{i}\left(\mathbf{k}_{j j_{1}}+\mathbf{k}_{j^{\prime} j_{1}^{\prime}}\right) \cdot \mathbf{r}\right]\left(\begin{array}{rrr}
K & K^{\prime} & K_{c} \\
Q & -Q^{\prime} & Q_{c}
\end{array}\right) \\
& \times \lambda_{Q_{c}}^{K_{c}}\left(\bar{K}, j, j_{1} ; \bar{K}^{\prime}, j^{\prime}, j_{1}^{\prime}\right) D_{\bar{K} \bar{K}^{\prime}}^{K K_{c} K^{\prime}} \\
& \times\left(F_{1}, F_{1}^{\prime} ; F_{2}, F_{2}^{\prime}\right) \varrho_{Q^{\prime}}^{K^{\prime}}\left(F_{2}, F_{2}^{\prime} ;-\right),(\mathrm{A} 13 \mathrm{~d}) \\
& D_{\bar{K} \bar{K}^{\prime}}^{K K^{\prime}}\left(F_{1}, F_{1}^{\prime} ; F_{2}, F_{2}^{\prime}\right)=(-1)^{K+K_{c}+K^{\prime}+\bar{K}+\bar{K}^{\prime}+K_{e}+K_{e}^{\prime}+K_{e}^{\prime \prime}+K_{n}} \\
& \times\left\{K, K_{c}, K^{\prime}, K_{e}, K_{n}^{2}, K_{e}^{\prime \prime}, F_{1}, F_{1}^{\prime}, F_{2}, F_{2}^{\prime}\right\}\left\{\begin{array}{lll}
K & K^{\prime} & K_{c} \\
K_{\mathrm{e}}^{\prime \prime} & K_{e} & K_{n}
\end{array}\right\} \\
& \times\left\{\begin{array}{lll}
\bar{K} & \bar{K}^{\prime} & K_{c} \\
K_{\mathrm{e}}^{\prime \prime} & K_{e} & K_{\mathrm{e}}^{\prime}
\end{array}\right\} C_{K_{e}^{\prime} \bar{K}}^{K_{e}}\left(J_{1}, J_{3}\right) C_{K_{e}^{\prime \prime} \bar{K}^{\prime}}^{K^{\prime}}\left(J_{3}, J_{2}\right) \\
& \times\left\{\begin{array}{lll}
K_{e} & K_{n} & K \\
J_{1} & I & F_{1} \\
J_{1} & I & F_{1}^{\prime}
\end{array}\right\}\left\{\begin{array}{lll}
K_{e}^{\prime \prime} & K_{n} & K^{\prime} \\
J_{2} & I & F_{2} \\
J_{2} & I & F_{2}^{\prime}
\end{array}\right\} \\
& \mathbf{k}_{i j}=\mathbf{k}_{i}-\mathbf{k}_{j} \text {, and } \\
& \lambda_{Q_{c}}^{K_{c}}\left(K, j, j_{1} ; K^{\prime}, j^{\prime}, j_{1}^{\prime}\right)=(-1)^{K+K^{\prime}+Q_{c}}\left\{K_{c}\right\}\left(\begin{array}{ccc}
K & K^{\prime} & K_{c} \\
Q & Q^{\prime} & -Q_{c}
\end{array}\right) \\
& \times \varepsilon_{Q}^{K}\left(j, j_{1}\right) \varepsilon_{Q^{\prime}}^{K^{\prime}}\left(j^{\prime}, j_{1}^{\prime}\right)
\end{aligned}
$$

$\tau=\int_{-\infty}^{\infty} d t \psi(t)$

There is no summation over $J_{1}$ and $J_{2}$ in (A13c, A13e).

We now consider the free evolution of the density matrix. The multipoles of the ground and excited states evolve as [21]

$$
\begin{aligned}
& \dot{\varrho}_{Q}^{K}\left(G, G^{\prime}\right)=-i \omega_{G G^{\prime}} \varrho_{Q}^{K}\left(G, G^{\prime}\right)+\Gamma^{(K)}\left(H, H^{\prime} ; G, G^{\prime}\right) \\
& \times \varrho_{Q}^{K}\left(H, H^{\prime}\right), \\
& \dot{\varrho}_{Q}^{K}\left(H, H^{\prime}\right)=-\left(\Gamma+\mathrm{i} \omega_{H H^{\prime}}\right) \varrho_{Q}^{K}\left(H, H^{\prime}\right), \\
& \text { where }
\end{aligned}
$$

$$
\begin{aligned}
\Gamma^{(K)}\left(H, H^{\prime}, G, G^{\prime}\right)= & (-1)^{H+K+G^{\prime}+1}\left\{H, H^{\prime}\right\}\left\{\begin{array}{lll}
H & H^{\prime} & K \\
G^{\prime} & G & 1
\end{array}\right\} \\
& \times \Gamma\left(H, H^{\prime} ; G, G^{\prime}\right), \\
\Gamma\left(H, H^{\prime} ; G, G^{\prime}\right)= & (4 / 3 \hbar)\left(\omega_{H G} \omega_{H^{\prime} G^{\prime}} / c^{2}\right)^{3 / 2}\left\{H^{-1}, H^{\prime-1}\right\} \\
& \times p_{G H}\left(p_{G^{\prime} H^{\prime}}\right)^{*},
\end{aligned}
$$

and $p_{G H}$ is a reduced element of the dipole moment operator.

In the free evolution period, there are generally nonzero initial values for density matrix elements $\varrho_{Q}^{K}\left(G, G^{\prime}\right)$, $\varrho_{Q}^{K}(G, H), \varrho_{Q}^{K}\left(H, H^{\prime}\right)$. Then, in a time $\Gamma^{-1}$, the optical coherence $\varrho_{Q}^{K}(G, H)$ decays and the excited state repopulates the ground state. When the processes of radiative decay are completed, $\Gamma\left(t-t_{i}\right) \gg 1$, one finds

$$
\begin{aligned}
& \varrho_{Q}^{K}\left(G, G^{\prime} ; t\right)=\exp \left[-\mathrm{i} \omega_{G G^{\prime}}\left(t-t_{i}\right)\right]\left[\varrho_{Q}^{K}\left(G, G^{\prime} ;+\right)\right. \\
& -(-1)^{H^{\prime}+G^{\prime}+K}\left\{J^{\prime 2}, G, G^{\prime}, H, H^{\prime}\right\} \\
& \times\left\{\begin{array}{lll}
H & H^{\prime} & K \\
G^{\prime} & G & 1
\end{array}\right\}\left\{\begin{array}{lll}
J & G & I \\
H & J^{\prime} & 1
\end{array}\right\}\left\{\begin{array}{lll}
J & G^{\prime} & I \\
H^{\prime} & J^{\prime} & 1
\end{array}\right\} \\
& \times\left[1-\mathrm{i}\left(\omega_{G G^{\prime}}-\omega_{H H^{\prime}}\right) / \Gamma\right]^{-1} \\
& \left.\times \varrho_{Q}^{K}\left(H, H^{\prime} ;+\right)\right] .
\end{aligned}
$$

If one considers the evolution of the spatially nonhomogeneous part of the density matrix (spatial harmonics) it is usually necessary to include Doppler shift terms in the energy denominator in (A18). This shift is different for different harmonics; however, for atoms cooled below the Doppler limit of laser cooling, owing to inequality (5), these shifts can be neglected.

By piecing together the solutions (A13) and (A18) one can construct the ground state density matrix induced by the ultrafast pulses. To monitor the spatial harmonics of the ground state density matrix one can scatter the probe pulse (12) from the medium. The pulse induces a polarization $\mathscr{P}$ having frequencies corresponding to the different hyperfine transitions and wave vectors combined from the probe field $\mathbf{k}_{\mathrm{p}}$ and those corresponding to the different harmonics of the ground state density matrix which were prepared before the probe field's action. The circular components of the positive frequency part of the polarization are given by

$$
\mathscr{P}_{q}=(-1)^{H-G} 3^{-1 / 2}\left[p_{H G} \varrho_{q}^{1}(G, H)\right]^{*},
$$


where $\varrho_{q}^{1}(G, H)$ is determined by an equation [23] analogous to $(\mathrm{A} 5 \mathrm{c})$ with the probe as a driving field, i.e.,

$$
\begin{aligned}
\dot{\varrho}_{q}^{1}(G, H)= & -\left(\Gamma / 2-\mathrm{i} \omega_{H G}\right) \varrho_{q}^{1}(G, H) \\
& +\mathrm{i} \mathrm{e}^{\mathrm{i}\left(\Omega_{\mathrm{p} t}-\mathbf{k}_{\mathrm{p}} \cdot \mathrm{r}\right)}\left(\chi_{H G^{\prime}}^{(p)}\right)^{*}(-1)^{G+G^{\prime}}\left(\varepsilon_{q^{\prime}}^{(p)}\right)^{*} \\
& \times \Lambda_{Q_{q}^{\prime} q}^{K 1}\left(H, G, G^{\prime}\right) \varrho_{Q}^{K}\left(G, G^{\prime}\right) .
\end{aligned}
$$

In contrast to (A5c), (A20) is written using the basis (A1), incorporates decay terms, and neglects any contribution from the excited state density matrix elements since they vanish at the time of application of the probe pulse. For a probe pulse with real envelope function $\psi_{\mathrm{p}}(t)$, $\psi_{\mathrm{p}}(0)=1$ and duration $\tau_{\mathrm{p}}=\int_{-\infty}^{\infty} d t \psi_{\mathrm{p}}(t)$ it follows that,
for

$t-t_{\mathrm{p}} \approx \Gamma^{-1} \gg \tau_{\mathrm{p}}$,

the polarization component $\mathscr{P}_{\mathrm{s}}=\mathscr{P}_{q} \cdot\left(\varepsilon_{q}^{(s)}\right)^{*}$ in the direction $\varepsilon_{\mathrm{s}}=(-1)^{q} \varepsilon_{-q}^{(s)} \varepsilon_{q}$ is given by

$$
\begin{aligned}
\mathscr{P}_{\mathrm{s}}= & \mathrm{i}(-1)^{H+G^{\prime}+K} \frac{\mathscr{E}_{\mathrm{p}}}{2}\left|p_{J^{\prime} J}\right|^{2} \tau_{\mathrm{p}} \\
& \times \exp \left[-\left(\Gamma / 2+\mathrm{i} \omega_{H G}\right)\left(t-t_{\mathrm{p}}\right)-\mathrm{i} \Omega_{\mathrm{p}} t_{\mathrm{p}}\right]\left\{H^{2}, G, G^{\prime}\right\} \\
& \times\left\{\begin{array}{lll}
1 & 1 & K \\
G & G^{\prime} & H
\end{array}\right\}\left\{\begin{array}{lll}
J^{\prime} & H & I \\
G & J & 1
\end{array}\right\}\left\{\begin{array}{lll}
J^{\prime} & H & I \\
G^{\prime} & J & 1
\end{array}\right\} \\
& \times \varepsilon_{Q}^{K}(p, s)\left\langle\mathrm{e}^{\mathrm{i} \mathbf{k}_{\mathrm{p}} \cdot \mathrm{r}_{\mathrm{p}}} \varrho_{Q}^{K}\left(G^{\prime}, G ; t_{\mathrm{p}}\right)\right\rangle,
\end{aligned}
$$

where $\mathbf{r}_{\mathrm{p}}=\mathbf{r}-\mathbf{v}\left(t-t_{\mathrm{p}}\right)$ is the atomic radius vector at the time of the probe pulse and $\langle\ldots\rangle$ indicates an average over velocities.

We now choose a specific field geometry in which $\mathbf{k}_{1}=-\mathbf{k}_{2}=\mathbf{k}_{\mathrm{p}}=k \hat{\mathbf{z}}$. For the backward scattering off the grating (4) prepared by the one pumping pulse, i.e., for the MGFID, one gets from (A13a, b) and (A18)

$$
\begin{aligned}
& \mathscr{P}_{\mathrm{s}}=\mathrm{i} \mathscr{E}_{\mathrm{p}}\left|p_{J^{\prime} J}\right|^{2} \chi_{J^{\prime} J}^{(2)}\left[\chi_{J^{\prime} J}^{(1)}\right]^{*} \tau_{\mathrm{p}} \tau^{2} \exp \left[-\Gamma / 2\left(t-t_{\mathrm{p}}\right)-\mathrm{i} k z-\mathrm{i} \Omega_{\mathrm{p}} t_{\mathrm{p}}\right] \\
& \times\left\langle\exp \left[i k v\left(t+t_{\mathrm{p}}\right)\right]\right\rangle P_{H G}\left(t_{\mathrm{p}}\right) \\
& \times \exp \left[-i \omega_{H G}\left(t-t_{\mathrm{p}}\right)\right] \text {, } \\
& P_{H G}\left(t_{\mathrm{p}}\right)=P_{H G}^{\mathrm{f}} \sum_{n} P_{H G}^{(n)} \exp \left(\mathrm{in} B t_{\mathrm{p}} / 2\right), \\
& P_{H G}^{(n)}=\tilde{P}_{H G}^{(n)} / P_{H G}^{\mathrm{f}}, \\
& P_{H G}^{\mathrm{f}}=\left(\sum_{n}\left|\tilde{P}_{H G}^{(n)}\right|^{2}\right)^{1 / 2}, \\
& \tilde{P}_{H G}^{(n)}=(-1)^{H+G^{\prime}+K+K^{\prime}}(1 / 4) \delta_{n, \tilde{\omega}\left(G, G^{\prime}\right)}\left\{K^{\prime-1}, H^{2}, G, G^{\prime}\right\} \\
& \times\left\{\begin{array}{lll}
1 & 1 & K \\
G & G^{\prime} & H
\end{array}\right\}\left\{\begin{array}{lll}
J^{\prime} & H & I \\
G & J & 1
\end{array}\right\}\left\{\begin{array}{lll}
J^{\prime} & H & I \\
G^{\prime} & J & 1
\end{array}\right\} \\
& \times \widetilde{C}_{K^{\prime} \bar{K}}^{K}\left(G^{\prime}, G, G^{\prime \prime}, G^{\prime \prime \prime}\right) \lambda_{Q^{\prime}}^{K^{\prime}}(K, p, s ; \bar{K}, 2,1) \\
& \times Q_{Q}^{K},\left(G^{\prime \prime}, G^{\prime \prime \prime} ; 0\right),
\end{aligned}
$$

$$
\begin{aligned}
& \tilde{C}_{K^{\prime} \bar{K}}^{K}\left(G, G^{\prime} ; G^{\prime \prime}, G^{\prime \prime \prime}\right)=\bar{C}_{K^{\prime}}^{K}\left(G, G^{\prime} ; G^{\prime \prime}, G^{\prime \prime \prime}\right) \\
& -(-1)^{H^{\prime}+G^{\prime}+K}\left\{J^{\prime 2}, G, G^{\prime}, H, H^{\prime}\right\} \\
& \times\left\{\begin{array}{ccc}
H & H^{\prime} & K \\
G^{\prime} & G & 1
\end{array}\right\}\left\{\begin{array}{lll}
J & G & I \\
H & J^{\prime} & 1
\end{array}\right\} \\
& \times\left\{\begin{array}{lll}
J & G^{\prime} & I \\
H^{\prime} & J^{\prime} & 1
\end{array}\right\} \\
& \times\left[1-\mathrm{i}\left(\omega_{G G^{\prime}}-\omega_{H H^{\prime}}\right) / \Gamma\right]^{-1} \\
& \times \bar{C}_{K^{\prime} \bar{K}}^{K}\left(H, H^{\prime} ; G^{\prime \prime}, G^{\prime \prime \prime}\right),
\end{aligned}
$$

where $B$ is the hyperfine splitting constant of the ground state, $\delta_{i k}$ is the Kronecker $\delta$ and $\varrho_{Q^{\prime}}^{K^{\prime}}\left(G, G^{\prime} ; 0\right)$ is the density matrix before the first excitation pulse. For an equilibrium initial state

$\varrho_{Q}^{K \prime},\left(G, G^{\prime} ; 0\right)=N\left\{G, J^{-2}, I^{-2}\right\} \delta_{K 0} \delta_{Q 0} \delta_{G G \prime}$,

where $N$ is the density of atoms, one arrives at (14) of Sect. 1.

Consider now the MGE. For the backward scattered radiation off the grating (6), prepared by two pulses delayed in time, one obtains

$$
\begin{aligned}
\mathscr{P}_{\mathrm{s}}= & \mathrm{i} \mathscr{E}_{\mathrm{p}}\left|p_{J^{\prime} J} \chi_{J^{\prime} J}^{(1)} \chi_{J^{J} J}^{(2)}\right|^{2} \chi_{J^{\prime} J}^{(2)}\left[\chi_{J^{\prime} J}^{(1)} *^{*} \tau_{\mathrm{p}} \tau^{6}\right. \\
& \times \exp \left[-\Gamma / 2\left(t-t_{\mathrm{p}}\right)-\mathrm{i} k z-\mathrm{i} \Omega_{\mathrm{p}} t_{\mathrm{p}}\right] \\
& \times\left\langle\exp \left[\mathrm{i} k v\left(t+t_{\mathrm{p}}-4 T\right)\right]\right\rangle \\
& \times P_{H G}\left(t_{\mathrm{p}}, T\right) \exp \left[-\mathrm{i} \omega_{H G}\left(t-t_{\mathrm{p}}\right)\right],
\end{aligned}
$$

$P_{H G}\left(t_{\mathrm{p}}, T\right)=P_{H G}^{\mathrm{e}} \sum_{n, m} P_{H G}^{(n, m)} \exp \left[i \frac{B}{2}\left(n T+m t_{\mathrm{p}}\right)\right]$,

$P_{H G}^{(n, m)}=\widetilde{P}_{H G}^{(n, m)} / P_{H G}^{\mathrm{e}}$,

$P_{H G}^{\mathrm{e}}=\left(\sum_{n, m}\left|\tilde{P}_{H G}^{(n, m)}\right|^{2}\right)^{1 / 2}$,

$\widetilde{P}_{H G}^{(n, m)}=(1 / 96)(-1)^{H+G^{\prime}+K+\bar{K}_{2}+K_{c}+K_{c}^{\prime}+Q_{2}} \delta_{n_{s} \tilde{\omega}\left(G^{\prime}, G\right)-\tilde{\omega}\left(G_{1}, G_{1}^{\prime}\right)}$

$$
\begin{aligned}
& \times \delta_{m, \tilde{\omega}\left(G, G^{\prime}\right)}\left\{K_{c}^{\prime}, H^{2}, G, G^{\prime}\right\}\left(\begin{array}{rrr}
K_{c} & K_{c}^{\prime} & K_{2} \\
Q_{c} & Q_{c}^{\prime} & -Q_{2}
\end{array}\right) \\
& \times \lambda_{Q_{c}}^{K_{c}}\left(\bar{K}, 2,1 ; \bar{K}_{1}, 2,1\right) \lambda_{Q_{c}^{\prime}}^{K_{c}^{\prime}}\left(K, p, s ; \bar{K}_{2}, 1,2\right) \\
& \times\left\{\begin{array}{lll}
K_{c} & K_{c}^{\prime} & K_{2} \\
\bar{K}_{2} & K_{1} & K
\end{array}\right\}\left\{\begin{array}{lll}
1 & 1 & K \\
G & G^{\prime} & H
\end{array}\right\}\left\{\begin{array}{lll}
J^{\prime} & H & I \\
G & J & 1
\end{array}\right\}\left\{\begin{array}{lll}
J^{\prime} & H & I \\
G^{\prime} & J & 1
\end{array}\right\} \\
& \times \tilde{D}_{\bar{K} \bar{K}_{1}}^{K K_{C} K_{1}}\left(G^{\prime}, G ; G_{1}, G_{1}^{\prime}\right) \tilde{C}_{K_{2} \bar{K}_{2}}^{K_{1}}\left(G_{1}, G_{1}^{\prime} ; G_{2}, G_{2}^{\prime}\right) \\
& \times \varrho_{Q_{2}}^{K_{2}}\left(G_{2}, G_{2}^{\prime} ; 0\right) \\
& \tilde{D}_{\bar{K}_{\bar{K}}}^{K_{K_{1}} K_{1}}\left(G, G^{\prime} ; G^{\prime \prime}, G^{\prime \prime \prime}\right)=D_{\bar{K}_{1} \bar{K}_{1}}^{K K_{1} K_{1}}\left(G, G^{\prime} ; G^{\prime \prime}, G^{\prime \prime \prime}\right) \\
& -(-1)^{H^{\prime}+G^{\prime}+K}\left\{J^{\prime 2}, G, G^{\prime}, H, H^{\prime}\right\}\left\{\begin{array}{lll}
H & H^{\prime} & K \\
G^{\prime} & G & 1
\end{array}\right\}\left\{\begin{array}{lll}
J & G & I \\
H & J^{\prime} & 1
\end{array}\right\} \\
& \times\left\{\begin{array}{lll}
J & G^{\prime} & I \\
H^{\prime} & J^{\prime} & 1
\end{array}\right\}\left[1-\mathrm{i}\left(\omega_{G G^{\prime}}-\omega_{H H^{\prime}}\right) / \Gamma\right]^{-1} \\
& \times D_{\bar{K} \bar{K}_{1}}^{K K_{c} K_{1}}\left(H, H^{\prime} ; G^{\prime \prime}, G^{\prime \prime \prime}\right) \text {. }
\end{aligned}
$$

One can also simplify these expressions for an initial equilibrium distribution (A24) to obtain (15) of the text. 


\section{References}

1. B. Dubetsky, P.R. Berman, T. Sleator: Phys. Rev. A 46, 2213 (1992)

2. H. Hatanaka, T. Hashi: J. Phys. Soc. Jpn. 39, 1139 (1975) A. Flusberg, T. Mossberg, R. Kachru, S.R. Hartman: Phys Rev. Lett. 41, 305 (1978)

3. B.Ya. Dubetsky, A.P. Kasantsev, V.P. Chebotayev, V.P. Yakovlev: Pis'ma Zh. Eksp. Teor. Fiz. 39, 531 (1984) [JETP Lett. 39, 649 (1984)]; J. Opt. Soc. Am. B 2, 1791 (1985)

4. O. Carnal, J. Mlynek: Phys. Rev. Lett. 66, 2689 (1991)

5. D.W. Keith, C.R. Ekstrom, Q.A. Turchette, D.E. Pritchard: Phys. Rev. Lett. 66, 2693 (1991)

6. R. Friedberg. S.R. Hartman: Laser Phys. 3, 526 (1993)

7. Ch.J. Bordé: Phys. Lett. A 140, 10 (1989)

8. M. Kasevich, S. Chu: Phys. Rev. Lett. 67, 181 (1991)

9. F. Riehle, Th. Kisters, A. Witte, J. Helmcke, Ch.J. Bordé: Phys. Rev. Lett. 67, 177 (1991)

10. Ye.V. Baklanov, B.Ya. Dubetsky, V.P. Chebotayev: Appl. Phys. 9, 171 (1976)

V.P. Chebotayev: Appl. Phys. 15, 219 (1978)

11. J.L. LeGouet, P.R. Berman: Phys. Rev. A 20, 1105 (1979)

12. Ye.V. Baklanov, B.Ya. Dubetsky, V.M. Semibalamut: Zh. Eksp. Teor. Fiz. 76, 482 (1979) [Sov. Phys.-JETP 49, 244 (1979)]

13. L.S. Vasilenko, N.M. Dyuba, M.N. Skvortsov, V.P. Chebotayev: Appl. Phys. 15, 319 (1978)

14. T.W. Mossberg, R. Kachru, E. Whittaker, S.R. Hartman: Phys. Rev. Lett. 43, 851 (1979)

15. R. Kachru, T.W. Mossberg, W. Whittaker, S.R. Hartman: Opt. Commun. 31, 223 (1979)

16. B. Dubetsky, P.R. Berman: Laser Phys. (in press)

17. Y. Castin, J. Dalibard, C. Cohen-Tannoudji: In Light-Induced Kinetic-Effects on Atoms, Ions and Molecules, ed. by L. Moi, S. Gozzini, C. Gabbannini, E. Arimondo, F. Strumin (ETS, Pisa 1991) pp. 5-24

18. For a general review of the use of optical coherent technique for carrying out rf spectroscopy, see D. Suter, J. Mlynek: In $A d-$ vances in Magnetic-and Optical Resonance, ed. by W.S. Warren, Vol. 16 (Academic New York 1992) p. 1, and references therein

19. A Messiah: Quantum Mechanics, Vol. II (Wiley, New York 1960) Appendix C. In Particular, the Winger-Eckart Theorem is taken as $\left\langle F, m\left|T_{q}^{k}\right| F^{\prime}, m^{\prime}\right\rangle=(-1)^{F^{\prime}-K+m}\left\langle F\left\|T^{k}\right\| F^{\prime}\right\rangle\left(\begin{array}{cc}F^{\prime} K & F \\ m^{\prime} q & -m\end{array}\right)$

20. M. Dumont, B. Decomps: J. Phys. (Paris) 29, 181 (1968)

M. Dumont: J. Phys. (Paris) 33, 971 (1972)

A. Omont: In Progress in Quantum Electronics, Vol. 5 (Pergamon, Oxford 1977) pp. 69-138

C. Feuillade, P.R. Berman: Phys. Rev. A 29, 1236 (1984)

M. Ducloy, D. Bloch: Phys. Rev. A 30, 3107 (1984)

K. Blum: Density Matrix Theory and Applications (Plenum, New York 1981)

B.W. Shore: The Theory of Coherent Atomic Excitation, Vol. 2 (Wiley Interscience, New York 1990) Sects. 21.9, 21.10

21. P.R. Berman: Phys. Rev. A 43, 1470 (1991)

22. P.R. Berman, G. Rogers, B. Dubetsky: Phys. Rev. A 48, 1506 (1993)

23. See $(\mathrm{Alc})$ in $[21]$ 